\title{
Role of Ubiquitin and SUMO in Intracellular Trafficking
}

\author{
Maria Sundvall $1,2,3 *$
}

\begin{abstract}
${ }^{1}$ Institute of Biomedicine, University of Turku, Turku, Finland.
${ }^{2}$ Western Cancer Centre FICAN West, Turku University Hospital, Turku, Finland.

${ }^{3}$ Department of Oncology and Radiotherapy, University of Turku, Turku, Finland.

*Correspondence: maria.sundvall@utu.fi
\end{abstract}

https://doi.org/10.21775/cimb.035.099

\begin{abstract}
Precise location of proteins at a given time within a cell is essential to convey specific signals and result in a relevant functional outcome. Small ubiquitin-like modifications, such as ubiquitin and SUMO, represent a delicate and diverse way to transiently regulate intracellular trafficking events of existing proteins in cells. Trafficking of multiple proteins is controlled reversibly by ubiquitin and/or SUMO directly or indirectly via regulation of transport machinery components. Regulation is dynamic and multilayered, involving active crosstalk and interdependence between post-translational modifications. However, in most cases regulation appears very complex, and the mechanistic details regarding how ubiquitin and SUMO control protein location in cells are not yet fully understood. Moreover, most of the findings still lack in vivo evidence in multicellular organisms.
\end{abstract}

\section{Posttranslational modifications in regulation of cellular processes}

\section{General principles of ubiquitination and SUMOylation}

Posttranslational modification (PTM) of proteins is a powerful, fast and often transient way to control the fate of existing proteins and cell behaviour. In addition to e.g. chemical groups, such as phosphate groups, proteins can be modified by small polypeptides, such as ubiquitin and SUMO. Ubiquitin and SUMO share a similar three-dimensional structure and are principally covalently linked at lysine $(\mathrm{K})$ residues of substrate proteins by a similar conjugation pathway (Hershko et al., 1998; Hay, 2013). Unlike ubiquitin, SUMO is preferentially attached at SUMO consensus sites $\Psi \mathrm{KxE}$ in substrates $\Psi$ =hydrophobic residue with high preference for I or $\mathrm{V}, \mathrm{x}=$ any amino acid) under steady state conditions, but under stress conditions in particular more nonconsensus sites are SUMOylated (Hendriks et al., 2016). Mammals express ubiquitously SUMO1, SUMO2 and SUMO3, whereas SUMO4 and SUMO5 are only expressed in some tissues and their functional role is unclear (Pichler et al., 2017). SUMO2 and SUMO3 are nearly identical and resemble approximately 50\% to SUMO1 (Geiss-Friedlander et al., 2007).

Precursors of ubiquitin and SUMO are processed to mature forms prior to conjugation and activated by an E1 enzyme in a reaction depending on ATP. Subsequently ubiquitin and SUMO are transferred to an E2 enzyme, and E3 ligase alone or with E2 ligates them to substrates by an isopeptide bond (Hershko et al., 1998; Hay, 2013). Modification is reversible and specific proteases can cleave ubiquitin and SUMO from substrates (Williamson et al., 2013; Kunz et al., 
2018). Proteins can be tagged by a single ubiquitin or SUMO either at one or at multiple lysines. In addition, modifications can exist as chains. Ubiquitin contains seven internal lysine residues (K6, K11, K27, K29, K33, K48, K63) that are involved in the formation of ubiquitin-ubiquitin polymers, known as polyubiquitin chains (Pickart et al., 2004; Heride et al., 2014). In addition, the linkage between the amino-terminal amino group of methionine on a ubiquitin can be conjugated with a target protein and the carboxy-terminal carboxy group of the incoming ubiquitin for linear chains (Walczak et al., 2012). SUMOs can also form polymeric chains through internal lysine residues (Geiss-Friedlander et al., 2007). Monoubiquitination and different chain types determine the fate of the modified protein (Piper et al., 2014). For example, K48 ubiquitin chains are considered classical signals for proteasomal degradation and K63 ubiquitin chains are linked to trafficking and DNA damage response (Pickart et al., 2004). Recently basic principles of the field have been challenged by e.g. discoveries of mixed polyubiquitin chains and ubiquitination of non-lysine residues (Piper et al., 2014). Less is known regarding the consequences of attachment of either single or many SUMO moieties. SUMO chains are at least implicated in recruitment of SUMO-targeted ubiquitin ligases (LallemandBreitenbach et al., 2008).

\section{Components, regulation and function of ubiquitin and SUMO machinery}

Multiple enzymes involved in ubiquitin conjugation have been recognized. The human ubiquitin machinery comprises a network including two ubiquitin E1 enzymes, approximately 40 ubiquitin E2s, and more than 600 E3 ubiquitin ligases in the human genome (Heride et al., 2014). Three major types of E3 ligases are really interesting new gene (RING) type E3s comprising most of human E3s, homologous to E6-AP carboxyl terminus (HECT) and RING-between-RING (RBR) E3s ligases (Deshaies et al., 2009; Rotin et al., 2009; Wenzel et al., 2012). Ubiquitin is cleaved by approximately 100 deubiquitinating enzymes (DUBs) (Williamson et al., 2013; Heride et al., 2014). Intriguingly, only one heterodimeric
E1 (Sae1/Aos1-Sae2/Uba2) and E2 (Ubc9) are known to be involved in SUMO conjugation (Hay, 2013). Three classes of SUMO E3s have been widely accepted and characterized including SP-RING Siz/PIAS ligases, RanBP2 and ZNF451 ligases (Geiss-Friedlander et al., 2007; Rytinki et al., 2009; Cappadocia et al., 2015). Both E2 and E3 can select substrates for SUMOylation, and spatial and temporal regulation of co-localization appears integral for substrate selection (Pichler et al., 2017). Cysteine proteases of the sentrinspecific protease (SENP) family members reverse SUMO conjugation in mammals (Kunz et al., 2018). Moreover, desumoylating isopeptides 1 and 2 and ubiquitin-specific protease-like 1 can deSUMOylate proteins (Shin et al., 2012; Schulz et al., 2012). Whereas ubiquitin machinery is widely expressed within a cell, components of SUMO conjugation pathway mainly localize at the nuclear pores and nucleus. Thus, most of SUMOylated substrates are nuclear proteins, although SUMO modified proteins outside of nucleus exist (GeissFriedlander et al., 2007).

Ubiquitination is regulated by extracellular stimuli including growth factors and cytokines, stress and cell cycle changes (Pickart et al., 2004; Heride et al., 2014). Different types of stress stimuli such as heat shock, hypoxia, reactive oxygen species, DNA damage and proteotoxic stress regulate the activity of SUMOylation machinery (Hietakangas et al., 2003; Shao et al., 2004; Bossis et al., 2006; Galanty et al., 2009; Morris et al., 2009; Seifert et al., 2015). Both ubiquitin and SUMO modifications can alter the function, activity, location and stability of their targets. Ubiquitin and SUMO are recognized by either ubiquitin-binding domains (UBD) or sumo-interacting domains (SIM), respectively, and serve as platforms for non-covalent protein-protein interactions (Seet et al., 2006). These domains have been identified in hundreds of proteins. PTMs are also involved in regulation of conjugation specificity and activation (Pichler et al., 2017). For example, SUMO-targeted ubiquitin ligase (STUbL) RNF4 contains multiple SIMs and a RING-domain to bind SUMOylated proteins and an E2 ubiquitinconjugation enzyme (Sun et al., 2007; Tatham et al., 2008). 


\section{Ubiquitin and SUMO as signals regulating membrane trafficking and endocytosis}

\section{General principles of membrane protein trafficking and endocytosis in cells}

Internalization and endocytosis of cell surface proteins including different receptors often occurs via the clathrin-dependent endocytic pathway. Cell surface receptors are clustered to pits coated with clathrin that pinch off of the membrane forming vesicles and early endosomes. From early endosomes cargo can be directed back to plasma membrane via recycling endosomes or destined to lysosomal degradation via late endosomes and multivesicular bodies (Mellman and Yarden, 2013). Also, other types of endocytic routes exist, including cholesterol-rich membrane structures, such as lipid rafts and caveolae (Barbieri et al., 2016). Several adaptor proteins and PTMs control these processes (Piper et al., 2014). Membrane protein trafficking and endocytosis system are tightly connected to protein homeostasis.

\section{Ubiquitin and SUMO in receptor internalization at the cell surface and in endocytic compartments}

Initial studies in yeast suggested that ubiquitin can function as a sorting signal regulating the internalization and endosomal targeting of cell surface receptors (Kölling et al., 1994; Hicke et al. 1996, Terrell et al., 1998). After that several studies in different systems and organisms have confirmed that ubiquitin is important regulator of endocytosis and its most critical functional role is likely at the sorting endosomes (Mellman and Yarden, 2013). Endocytosis of human receptor tyrosine kinases (RTKs) is suggested to be regulated by multimonoubiquitination and K63-polyubiquitination, and there is some controversy regarding the significance of specific ubiquitination type due to methodological challenges to address this complex regulatory system (Haglund et al., 2003; McCullough et al., 2004; Huang et al., 2006; Sundvall et al., 2008; Huang et al., 2013). During endocytosis the ubiquitin moieties of cargo are recognized by different endocytic adaptors and regulators via UBDs, such as Eps15, epsin and endosomal sortin complex required for transport (ESCRT) (Piper et al., 2014). Ubiquitin is also indirectly involved in control of endocytosis as components of endocytic machinery are actively regulated by ubiquitination (Piper et al., 2014).

SUMOylation has been implicated in the regulation of endocytic processes, although when compared to ubiquitin the evidence is less extensive, and more work is needed to make general conclusions. Nevertheless, endocytosis of kainate receptor GluR6 is regulated by SUMOylation and non-SUMOylated mutant of GluR6, GluR6$\mathrm{K} 886 \mathrm{R}$, is endocytosis-impaired due to unknown mechanisms (Martin et al., 2007). SUMOylation of Smoothened (Smo) promotes its localization at the cell surface (Ma et al., 2016). Intriguingly, mechanisticallySUMO interferes with efficient Smo ubiquitination by recruiting deubiquitinase UBPY/ USP8 in a SIM-dependent manner (Ma et al.,2016). SUMOylation regulates also cell surface expression and activity of VEGFR2 receptor tyrosine kinase (Zhou et al., 2018). VEGFR2-SUMO1 fusion protein but not SUMOylation defective mutant VEGFR2 accumulated at the Golgi suggesting that mechanistically SUMO regulates exocytosis of VEGFR (Zhou et al., 2018). SUMOylation can also indirectly regulate endocytosis. Components of endocytic machinery are modified and regulated by SUMO, such as CIN85 (Tossidou et al., 2012) and arrestin (Wyatt et al., 2011). Interestingly, also dynamin interacts with several members of the SUMOylation machinery (Mishra et al., 2004). Moreover, SUMO can be important for membrane binding of proteins. SUMOylation of PTEN at lysine 266 within CBR3 loop fosters binding of PTEN to plasma membrane via electrostatic interactions (Huang et al., 2012).

\section{Ubiquitin and SUMO as signals regulating nucleocytoplasmic shuttling and subnuclear targeting}

\section{General principles of nuclear import, subnuclear targeting and export}

Passage of proteins in and out of the nucleus through nuclear pore complexes is tightly regulated. 
In principle, many of the nuclear proteins contain nuclear localization signal (NLS) and nuclear export signal (NES) that facilitate trafficking via associations with karyopherins including importins and exportins (CRM1) together with Ran-GTP, respectively (Stewart M, 2007).

\section{Ubiquitin and SUMO in nuclear targeting and trapping}

Molecular mechanism by which ubiquitin machinery controls protein functions are very complex but, nevertheless, some evidence exists to suggest the role for ubiquitin as a signal controlling nucleocytoplasmic trafficking. Ubiquitination of p53 contributes to nuclear export, and although the regulation of p53 ubiquitination and trafficking has turned out to be very complicated and likely dependent of conditions, attachment of monoubiquitin in particular is suggested to play a role in trafficking (Lohrum et al., 2001; Li et al., 2003). Moreover, similar type of regulation has been suggested for another tumour suppressor protein, PTEN and NF-kB essential modulator NEMO (Huang et al., 2003; Trotman et al., 2007).

SUMO was initially discovered as a modifier of RanGap1 targeting it to nuclear pore complex (Matunis et al., 1996, Mahajan et al., 1997). Later SUMO has been implicated in both increasing and decreasing the nuclear accumulation of some proteins. Mechanistically, covalent linkage of SUMO may directly block protein interactions relevant for transport or generate SIM-mediated interaction platform facilitating or interfering with transport. SUMOylation is suggested to regulate nuclear import of full-length IGF receptor (Sehat et al., 2010). The levels of SUMOylation defective mutant IGFR are similar at the cell surface compared with wild type receptor, but the mutant receptor cannot translocate to nucleus unlike wild type (Sehat et al., 2010). Specifically IGFR is suggested to interact with RanBP2 at nuclear pores and that RanBP2 acts as an SUMO E3 ligase for IGFR (Pancham et al., 2015). SUMOylation may also increase the stability of IGFR (Pancham et al., 2015). Trafficking of SUMO machinery components is also under the control of SUMO. SUMOylation of Sae2 in the c-terminus within functional NLS efficiently increases nuclear accumulation (Truong et al., 2012).
SUMO has been suggested to regulate nuclear export of some proteins, including transcriptional repressor TEL, Kruppel-like transcription factor (KLF2), Serine hydroxylmethyltransferase 1 (SHMT1), PTEN, p53, ErbB4 and Notch (Wood et al., 2003; Du et al., 2008, Anderson et al., 2009; Bassi et al., 2013, Santiago et al., 2013, Knittle et al., 2017; Antila et al., 2018). SUMOylation adjacent to NES of KLF5 interferes with its interaction with nuclear export receptor CRM1 resulting in the inhibition of efficient export and increased accumulation in the nucleus $(\mathrm{Du}$ et al., 2008). ErbB4 RTK undergoes regulated intramembrane proteolysis (RIP) releasing an intracellular domain (ICD) that can translocate to nucleus and regulate transcription. PIAS3 catalysed SUMOylation within NES of ErbB4 increases the nuclear accumulation of a tyrosine phosphorylated ICD by altering the interaction with CRM1 (Sundvall et al., 2012; Knittle et al., 2017). SUMOylation deficient mutant of ErbB4 accumulates less in nucleus and cannot convey efficiently nuclear signalling (Knittle et al., 2017). Another receptor undergoing RIP, Notch, is also SUMOylated in the nucleus and SUMOylation increases nuclear accumulation of the ICD, but mechanisms resulting in accumulation remain to be elucidated (Antila et al., 2018). SUMOylation of SHMT1 increases nuclear accumulation and mutation of the SUMO motif prevents translocation to the nucleus due to unknown mechanisms (Anderson DD et al., 2009). Interestingly, same sites are also ubiquitinated with K63 polyubiquitin chains increasing stability in the nucleus (Anderson et al., 2012). SUMOylation of PTEN (at K254) is suggested to regulate the efficient nuclear accumulation and subsequently DNA damage response. When compared to wild type, SUMOylation deficient mutant of PTEN localizes less into the nucleus and cannot efficiently regulate homologous recombination (Bassi et al., 2013). Nucleocytoplasmic distribution of SUMOylation-deficient transcriptional repressor TEL also changes compared to wild type (Wood et al., 2003). However, the direct mechanism how SUMO regulates subcellular localization of PTEN or TEL is not clear (Wood et al., 2003; Bassi et al., 2013). On the contrary, SUMOylation of p53 stimulates its nuclear export by increasing the 
disassembly of p53 from the CRM1 in the cytosol (Santiago et al. 2013). Moreover, SUMOylation regulates nuclear export and intranuclear distribution of adenovirus E1B-55K protein (Kindsmüller et al., 2007). Altogether nuclear export and SUMOylation appear to be closely connected due to CRM1-mediated interaction of export complexes with SUMO E3 ligase RanBP2 (Ritterhoff et al., 2015) and SUMOylation also regulates nuclear transport via covalent modification of transport machinery (Rothenbusch et al., 2012).

\section{Regulation of subnuclear targeting by ubiquitin and SUMO}

Ubiquitin and SUMO system has been indicated in targeting proteins into certain subnuclear structures. Subnuclear targeting can direct proteins into locations essential for their functions, trap protein in locations where they are not available to regulate e.g. transcription or alter their susceptibility to regulators of stability. Both ubiquitin and SUMO are implicated in correct targeting of DNA repair factors to the sites of DNA damage (Ulrich, 2014). For example, BRCA1 targeting to double-strand breaks is regulated by K63-linked ubiquitination and SUMO and the process involves RNF4 (Galanty et al., 2009; Morris et al., 2009; Guzzo et al., 2012). PML bodies are subnuclear structures involved in regulation of transcription and host a lot of transcription factors and their regulators (Zhong et al., 2000a). PML is strongly SUMOylated and SUMOylation regulates the integrity of PML bodies and stability of PML as SUMOylation deficient mutant PML does not form nuclear bodies when expressed in PML null cells (Ishov et al., 1999; Müller et al., 1998; Zhong et al., 2000a,b). Several nuclear proteins are hosted in PML bodies and SIM-mediated interactions are thought to be important for assembly (Shen et al., 2006). SUMO E3 ligases and deSUMOlases have specific nuclear localizations and regulate substrate localization, but regulation is often not SUMO-dependent (Sachdev et al., 2001; Kotaja et al., 2002; Hietakangas et al., 2003). On the other hand, SUMO is suggested to be important in the regulation of subnuclear localization of Nuclear Factor of Activated T-cells (NFAT1) (Terui et al., 2004) or nucleolar localization of Proline-, glutamic acid- and leucine-rich protein 1 (PELP1) involved in ribosome biogenesis and regulation of transcription (Finkbeiner et al., 2011).

\section{The genetic evidence in vivo and the significance of ubiquitin and SUMO mediated regulation of trafficking in human diseases}

Genetic studies using targeted gene disruption in mice suggest that ubiquitin and SUMO pathways are essential, but a lot of redundancy is evident with many of the pathway components. For example, SUMO1 and SUMO3 knockout mice are viable, but SUMO2 knockout mice die during embryogenesis as well as Ubc9 knockout is lethal in mice (Nacerddine et al., 2005; Evdokimov et al., 2008; Zhang et al., 2008; Wang et al., 2014). Unfortunately, very little is known regarding genetic models of modification-deficient mutants and their phenotypes in vivo.

Deregulation of ubiquitin conjugation machinery and altered protein ubiquitination has been reported in diseases such as neurodegenerative diseases and cancer (Popovic et al., 2014). SUMOylation seems to be a general protective mechanism against the damage caused by stresses such as low oxygen and nutrient deprivation and may also protect neurons after stress and support the growth of cancer cells. Mutations targeting ubiquitin conjugation machinery, such as E3 ligases, and somatic mutations altering ubiquitin ligase binding and subsequent trafficking of targets, including deregulated endocytosis of RTKs, in cancer have been reported (Mellman and Yarden, 2013). SUMOylation seems to be up-regulated in cancer due to e.g. overexpression of the pathway components, such as Ubc9 and some E3 ligases (Seeler et al., 2017). Interestingly, it has been reported that the SUMOylation site is essential for leukaemic transformation mediated by PML-RARalpha in acute promyelocytic leukaemia (Zhu et al., 2005). Moreover, a germline variant of melanoma lineage-specific microphthalmia-associated transcription factor (MITF), MITF-E318K, increases predisposition to sporadic melanoma and renal cell carcinoma. This mutation disrupts SUMOylation site of MITF and results in increased transcriptional activity promoting tumourigenic properties in experimental models, but no changes 
in trafficking were observed (Bertolotto et al., 2011; Yokoyama et al., 2011). However, despite extensive sequencing efforts, the evidence is so far lacking for ubiquitin or SUMO binding sites to be systematically targeted by somatic mutations in cancers. Development and research efforts using drugs that specifically inhibit the conjugation pathway activity and components will help to understand the role of SUMO and non-proteolytic functions of Ubiquitin in different contexts.

\section{Conclusions}

Emerging evidence strongly implicates both ubiquitination and SUMOylation in the regulation of intracellular trafficking of several proteins at multiple levels. However, these regulation systems are highly dynamic, complex and complicated with several overlapping compensatory mechanisms and the possibility of targeting same residues with different PTMs, making it challenging to design conclusive studies. In most cases the precise convincing mechanistic insights on how this regulation of trafficking happens remain to be elucidated. In addition, the field is still lacking evidence supporting many general findings in vivo. Nevertheless, conceptually ubiquitin and SUMO have already emerged as powerful and potent regulators of essential cellular processes, including protein trafficking to control temporal and special protein functions, and these PTMs are definitely an important focus for future research.

\section{Acknowledgements}

The laboratory of the author has been supported by grants from the Academy of Finland, Sigrid Juselius Foundation, Finnish Medical Foundation Duodecim, Turku University Foundation, Paulo Foundation and Instrumentarium Science Foundation. The author has been supported by Pfizer, Novartis, Celgene, MSD (conference participation costs not related to the topic of this manuscript) and Roche (conference participation costs and consultant fee not related to the topic of this manuscript). Dr Johanna Ahlskog and Dr Anna Knittle are acknowledged for valuable comments regarding the manuscript.

\section{References}

Anderson, D.D., and Stover, P.J. (2009). SHMT1 and SHMT2 are functionally redundant in nuclear de novo thymidylate biosynthesis. PLOS ONE 4, e5839. https: / / doi.org/10.1371/journal.pone.0005839.

Anderson, D.D., Eom, J.Y., and Stover, P.J. (2012). Competition between sumoylation and ubiquitination of serine hydroxymethyltransferase 1 determines its nuclear localization and its accumulation in the nucleus. J. Biol. Chem. 287, 4790-4799. https://doi. org/10.1074/jbc.M111.302174.

Antila, C.J.M., Rraklli, V., Blomster, H.A., Dahlström, K.M., Salminen, T.A., Holmberg, J., Sistonen, L., and Sahlgren, C. (2018). Sumoylation of Notch1 represses its target gene expression during cell stress. Cell Death Differ. 25, 600-615. https://doi.org/10.1038/s41418-017-00026.

Barbieri, E., Di Fiore, P.P., and Sigismund, S. (2016). Endocytic control of signaling at the plasma membrane. Curr. Opin. Cell Biol. 39, 21-27. https://doi. org/10.1016/j.ceb.2016.01.012.

Bassi, C., Ho, J., Srikumar, T., Dowling, R.J., Gorrini, C., Miller, S.J., Mak, T.W., Neel, B.G., Raught, B., and Stambolic, V. (2013). Nuclear PTEN controls DNA repair and sensitivity to genotoxic stress. Science 341, 395-399. https://doi.org/10.1126/science.1236188.

Bertolotto, C., Lesueur, F., Giuliano, S., Strub, T., de Lichy, M., Bille, K., Dessen, P., d'Hayer, B., Mohamdi, H., Remenieras, A., et al. (2011). A SUMOylation-defective MITF germline mutation predisposes to melanoma and renal carcinoma. Nature 480, 94-98. https://doi. org/10.1038/nature 10539 .

Bossis, G., and Melchior, F. (2006). Regulation of SUMOylation by reversible oxidation of SUMO conjugating enzymes. Mol. Cell 21, 349-357.

Cappadocia, L., Pichler, A., and Lima, C.D. (2015). Structural basis for catalytic activation by the human ZNF451 SUMO E3 ligase. Nat. Struct. Mol. Biol. 22, 968-975. https://doi.org/10.1038/nsmb.3116.

Deshaies, R.J., and Joazeiro, C.A. (2009). RING domain E3 ubiquitin ligases. Annu. Rev. Biochem. 78, 399-434. https://doi.org/10.1146/annurev. biochem.78.101807.093809.

Du, J.X., Bialkowska, A.B., McConnell, B.B., and Yang, V.W. (2008). SUMOylation regulates nuclear localization of Krüppel-like factor 5. J. Biol. Chem. 283, 31991-32002. https://doi.org/10.1074/jbc.M803612200.

Evdokimov, E., Sharma, P., Lockett, S.J., Lualdi, M., and Kuehn, M.R. (2008). Loss of SUMO1 in mice affects RanGAP1 localization and formation of PML nuclear bodies, but is not lethal as it can be compensated by SUMO2 and SUMO3. J. Cell Sci. 121, 4106-4113.

Finkbeiner, E., Haindl, M., and Muller, S. (2011). The SUMO system controls nucleolar partitioning of a novel mammalian ribosome biogenesis complex. EMBO J. 30, 1067-1078. https://doi.org/10.1038/emboj.2011.33.

Galanty, Y., Belotserkovskaya, R., Coates, J., Polo, S., Miller, K.M., and Jackson, S.P. (2009). Mammalian SUMO E3-ligases PIAS1 and PIAS4 promote responses to DNA double-strand breaks. Nature 462, 935-939. https://doi.org/10.1038/nature08657. 
Geiss-Friedlander, R., and Melchior, F. (2007). Concepts in sumoylation: a decade on. Nat. Rev. Mol. Cell Biol. 8, 947-956.

Guzzo, C.M., Berndsen, C.E., Zhu, J., Gupta, V., Datta, A., Greenberg, R.A., Wolberger, C., and Matunis, M.J. (2012). RNF4-dependent hybrid SUMO-ubiquitin chains are signals for RAP80 and thereby mediate the recruitment of BRCA1 to sites of DNA damage. Sci. Signal. 253, ra88. doi:10.1126/scisignal.2003485.

Haglund, K., Sigismund, S., Polo, S., Szymkiewicz, I., Di Fiore, P.P., and Dikic, I. (2003). Multiple monoubiquitination of RTKs is sufficient for their endocytosis and degradation. Nat. Cell Biol. 5, 461-466. https://doi.org/10.1038/ncb983.

Hay, R.T. (2013). Decoding the SUMO signal. Biochem. Soc. Trans. 41, 463-473. https://doi.org/10.1042/ BST20130015.

Hendriks, I.A., and Vertegaal, A.C. (2016). A comprehensive compilation of SUMO proteomics. Nat. Rev. Mol. Cell Biol. 17, 581-595. https://doi.org/10.1038/ nrm.2016.81.

Hendriks, I.A., Schimmel, J., Eifler, K., Olsen, J.V., and Vertegaal, A.C. (2015). Ubiquitin-specific protease 11 (USP11) deubiquitinates hybrid small ubiquitin-like modifier (SUMO)-ubiquitin chains to counteract RING finger protein 4 (RNF4). J. Biol. Chem. 290, 1552615537. https://doi.org/10.1074/jbc.M114.618132.

Heride, C., Urbé, S., and Clague, M.J. (2014). Ubiquitin code assembly and disassembly. Curr. Biol. 24, R215-20. https://doi.org/10.1016/j.cub.2014.02.002.

Hershko, A., and Ciechanover, A. (1998). The ubiquitin system. Annu. Rev. Biochem. 67, 425-479. https://doi. org/10.1146/annurev.biochem.67.1.425.

Hicke, L., and Riezman, H. (1996). Ubiquitination of a yeast plasma membrane receptor signals its ligand-stimulated endocytosis. Cell 84, 277-287.

Hietakangas, V., Ahlskog, J.K., Jakobsson, A.M., Hellesuo, M., Sahlberg, N.M., Holmberg, C.I., Mikhailov, A., Palvimo, J.J., Pirkkala, L., and Sistonen, L. (2003). Phosphorylation of serine 303 is a prerequisite for the stress-inducible SUMO modification of heat shock factor 1. Mol. Cell. Biol. 23, 2953-2968.

Huang, F., Kirkpatrick, D., Jiang, X., Gygi, S., and Sorkin, A. (2006). Differential regulation of EGF receptor internalization and degradation by multiubiquitination within the kinase domain. Mol. Cell 21, 737-748.

Huang, F., Zeng, X., Kim, W., Balasubramani, M., Fortian, A., Gygi, S.P., Yates, N.A., and Sorkin, A. (2013). Lysine 63-linked polyubiquitination is required for EGF receptor degradation. Proc. Natl. Acad. Sci. U.S.A. 110, 15722-15727. https://doi.org/10.1073/ pnas. 1308014110.

Huang, J., Yan, J., Zhang, J., Zhu, S., Wang, Y., Shi, T., Zhu, C., Chen, C., Liu, X., Cheng, J., et al. (2012). SUMO1 modification of PTEN regulates tumorigenesis by controlling its association with the plasma membrane. Nat. Commun. 3, 911. https://doi.org/10.1038/ ncomms 1919 .

Huang, T.T., Wuerzberger-Davis, S.M., Wu, Z.H., and Miyamoto, S. (2003). Sequential modification of NEMO/IKKgamma by SUMO-1 and ubiquitin mediates NF-kappaB activation by genotoxic stress. Cell $115,565-576$.
Ishov, A.M., Sotnikov, A.G., Negorev, D., Vladimirova, O.V., Neff, N., Kamitani, T., Yeh, E.T., Strauss, J.F., and Maul, G.G. (1999). PML is critical for ND10 formation and recruits the PML-interacting protein daxx to this nuclear structure when modified by SUMO-1. J. Cell Biol. 147, 221-234.

Kindsmüller, K., Groitl, P., Härtl, B., Blanchette, P., Hauber, J., and Dobner, T. (2007). Intranuclear targeting and nuclear export of the adenovirus E1B-55K protein are regulated by SUMO1 conjugation. Proc. Natl. Acad. Sci. U.S.A. 104, 6684-6689.

Knittle, A.M., Helkkula, M., Johnson, M.S., Sundvall, M., and Elenius, K. (2017). SUMOylation regulates nuclear accumulation and signaling activity of the soluble intracellular domain of the ErbB4 receptor tyrosine kinase. J. Biol. Chem. 292, 19890-19904.

Kölling, R., and Hollenberg, C.P. (1994). The ABCtransporter Ste6 accumulates in the plasma membrane in a ubiquitinated form in endocytosis mutants. EMBO J. 13, 3261-3271.

Kotaja, N., Karvonen, U., Jänne, O.A., and Palvimo, J.J. (2002). PIAS proteins modulate transcription factors by functioning as SUMO-1 ligases. Mol. Cell. Biol. 22: 5222-5234.

Kunz, K., Piller, T., and Müller, S. (2018). SUMO-specific proteases and isopeptidases of the SENP family at a glance. J. Cell. Sci. 131, jcs211904.

Lallemand-Breitenbach, V., Jeanne, M., Benhenda, S., Nasr, R., Lei, M., Peres, L., Zhou, J., Zhu, J., Raught, B., and de Thé, H. (2008). Arsenic degrades PML or PMLRARalpha through a SUMO-triggered RNF4/ubiquitinmediated pathway. Nat. Cell Biol. 10, 547-555. https:// doi.org/10.1038/ncb1717.

Li, M., Brooks, C.L., Wu-Baer, F., Chen, D., Baer, R., and $\mathrm{Gu}$, W. (2003). Mono- versus polyubiquitination: differential control of p53 fate by Mdm2. Science 302, 1972-1975. https://doi.org/10.1126/science.1091362.

Lohrum, M.A., Woods, D.B., Ludwig, R.L., Bálint, E., and Vousden, K.H. (2001). C-terminal ubiquitination of p53 contributes to nuclear export. Mol. Cell. Biol. 21, 85218532. https://doi.org/10.1128/MCB.21.24.85218532.2001.

Ma, G., Li, S., Han, Y., Li, S., Yue, T., Wang, B., and Jiang, J. (2016). Regulation of smoothened trafficking and hedgehog signaling by the SUMO pathway. Dev. Cell 39, 438-451.

Mahajan, R., Delphin, C., Guan, T., Gerace, L., and Melchior, F. (1997). A small ubiquitin-related polypeptide involved in targeting RanGAP1 to nuclear pore complex protein RanBP2. Cell 88, 97-107.

Martin, S., Nishimune, A., Mellor, J.R., and Henley, J.M. (2007). SUMOylation regulates kainate-receptormediated synaptic transmission. Nature 447, 321-325.

Matunis, M.J., Coutavas, E., and Blobel, G. (1996). A novel ubiquitin-like modification modulates the partitioning of the Ran-GTPase-activating protein RanGAP1 between the cytosol and the nuclear pore complex, J. Cell Biol. 135, 1457-1470.

McCullough, J., Clague, M.J., and Urbe, S. (2004). AMSH is an endosome-associated ubiquitin isopeptidase. J. Cell. Biol. 166, 487-492. 
Mellman, I., and Yarden, Y. (2013). Endocytosis and cancer. Cold Spring Harb. Perspect. Biol. 5, a016949. https:// doi.org/10.1101/cshperspect.a016949.

Mishra, R.K., Jatiani, S.S., Kumar, A., Simhadri, V.R., Hosur, R.V., and Mittal, R. (2004). Dynamin interacts with members of the sumoylation machinery. J. Biol. Chem. 279, 31445-31454. https://doi.org/10.1074/jbc. M402911200.

Morris, J.R., Boutell, C., Keppler, M., Densham, R., Weekes, D., Alamshah, A., Butler, L., Galanty, Y., Pangon, L., Kiuchi, T., et al. (2009). The SUMO modification pathway is involved in the BRCA1 response to genotoxic stress. Nature 462, 886-890. https://doi.org/10.1038/ nature08593.

Müller, S., Matunis, M.J., and Dejean, A. (1998). Conjugation with the ubiquitin-related modifier SUMO-1 regulates the partitioning of PML within the nucleus. EMBO J 17, 61-70.

Nacerddine, K., Lehembre, F., Bhaumik, M., Artus, J., Cohen-Tannoudji, M., Babinet, C., Pandolfi, P.P., and Dejean, A. (2005). The SUMO pathway is essential for nuclear integrity and chromosome segregation in mice. Dev. Cell 9, 769-779.

Packham, S., Warsito, D., Lin, Y., Sadi, S., Karlsson, R., Sehat, B., and Larsson, O. (2015). Nulear translocation of IGF-1R via $p 150$ Glued and an importin- $\beta /$ RanBP2dependent pathway in cancer cells. Oncogene 34: 2227-2238.

Pickart, C.M., and Fushman, D. (2004). Polyubiquitin chains: polymeric protein signals. Curr. Opin. Chem. Biol. 8, 610-616.

Pichler, A., Fatouros, C., Lee, H., and Eisenhardt, N. (2017). SUMO conjugation - a mechanistic view. Biomol. Concepts 8, 13-36. https://doi.org/10.1515/bmc2016-0030.

Piper, R.C., Dikic, I., and Lukacs, G.L. (2014). Ubiquitindependent sorting in endocytosis. Cold Spring Harb. Perspect. Biol. 6, a016808. https://doi.org/10.1101/ cshperspect.a016808.

Popovic, D., Vucic, D., and Dikic, I. (2014). Ubiquitination in disease pathogenesis and treatment. Nat. Med. 20, 1242-1253. https://doi.org/10.1038/nm.3739.

Ritterhoff, T., Das, H., Hofhaus, G., Schröder, R.R., Flotho, A., and Melchior, F. (2016). The RanBP2/ RanGAP1*SUMO1/Ubc9 SUMO E3 ligase is a disassembly machine for Crm1-dependent nuclear export complexes. Nat. Commun. 7, 11482. https://doi. org/10.1038/ncomms11482.

Rothenbusch, U., Sawatzki, M., Chang, Y., Caesar, S., and Schlenstedt, G. (2012). Sumoylation regulates Kap114mediated nuclear transport. EMBO J. 31, 2461-2472. https://doi.org/10.1038/emboj.2012.102.

Rotin, D., and Kumar, S. (2009). Physiological functions of the HECT family of ubiquitin ligases. Nat. Rev. Mol. Cell Biol. 10, 398-409. https://doi.org/10.1038/nrm2690.

Rytinki, M.M., Kaikkonen, S., Pehkonen, P., Jääskeläinen, T., and Palvimo, J.J. (2009). PIAS proteins: pleiotropic interactors associated with SUMO. Cell. Mol. Life Sci. 66, 3029-3041. https://doi.org/10.1007/s00018-0090061-z.

Sachdev, S., Bruhn, L., Sieber, H., Pichler, A., Melchior, F., and Grosschedl, R. (2001). PIASy, a nuclear matrixassociated SUMO E3 ligase, represses LEF1 activity by sequestration into nuclear bodies. Genes Dev. 15, 3088-3103.

Santiago, A., Li, D., Zhao, L.Y., Godsey, A., and Liao, D. (2013). p53 SUMOylation promotes its nuclear export by facilitating its release from the nuclear export receptor CRM1. Mol. Biol. Cell 24, 2739-2752. https:// doi.org/10.1091/mbc.E12-10-0771.

Schulz, S., Chachami, G., Kozaczkiewicz, L., Winter, U., Stankovic-Valentin, N., Haas, P., Hofmann, K., Urlaub, H., Ovaa, H., Wittbrodt, J., et al. (2012). Ubiquitinspecific protease-like 1 (USPL1) is a SUMO isopeptidase with essential, non-catalytic functions. EMBO Rep. 13, 930-938. https://doi.org/10.1038/embor.2012.125.

Seeler, J.S., and Dejean, A. (2017). SUMO and the robustness of cancer. Nat. Rev. Cancer 17, 184-197. https://doi.org/10.1038/nrc.2016.143.

Seet, B.T., Dikic, I., Zhou, M.M., and Pawson, T. (2006). Reading protein modifications with interaction domains. Nat. Rev. Mol. Cell Biol. 7, 473-483.

Sehat, B., Tofigh, A., Lin, Y., Trocmé, E., Liljedahl, U., Lagergren, J., and Larsson, O. (2010). SUMOylation mediates the nuclear translocation and signaling of the IGF-1 receptor. Sci. Signal. 3, ra10. https://doi. org/10.1126/scisignal.2000628.

Seifert, A., Schofield, P., Barton, G.J., and Hay, R.T. (2015). Proteotoxic stress reprograms the chromatin landscape of SUMO modification. Sci. Signal. 8, rs7. https://doi. org/10.1126/scisignal.aaa2213.

Shao, R., Zhang, F.P., Tian, F., Anders Friberg, P., Wang, X., Sjöland, H., and Billig, H. (2004). Increase of SUMO-1 expression in response to hypoxia: direct interaction with HIF-1alpha in adult mouse brain and heart in vivo. FEBS Lett. 569, 293-300. https://doi.org/10.1016/j. febslet.2004.05.079.

Shen, T.H., Lin, H.K., Scaglioni, P.P., Yung, T.M., and Pandolfi, P.P. (2006). The mechanisms of PML-nuclear body formation. Mol. Cell 24, 331-339.

Shin, E.J., Shin, H.M., Nam, E., Kim, W.S., Kim, J.H., Oh, B.H., and Yun, Y. (2012). DeSUMOylating isopeptidase: a second class of SUMO protease. EMBO Rep. 13, 339-346. https://doi.org/10.1038/embor.2012.3.

Stewart, M. (2007). Molecular mechanism of the nuclear protein import cycle. Nat. Rev. Mol. Cell Biol. 8, 195-208.

Sun, H., Leverson, J.D., and Hunter, T. (2007). Conserved function of RNF4 family proteins in eukaryotes: targeting a ubiquitin ligase to SUMOylated proteins. EMBO J. 26, 4102-4112.

Sundvall, M., Korhonen, A., Paatero, I., Gaudio, E., Melino, G., Croce, C.M., Aqeilan, R.I., and Elenius, K. (2008). Isoform-specific monoubiquitination, endocytosis, and degradation of alternatively spliced ErbB4 isoforms. Proc. Natl. Acad. Sci. U.S.A. 105, 4162-4167. https:// doi.org/10.1073/pnas.0708333105.

Sundvall, M., Korhonen, A., Vaparanta, K., Anckar, J., Halkilahti, K., Salah, Z., Aqeilan, R.I., Palvimo, J.J., Sistonen, L., and Elenius, K. (2012). Protein inhibitor of activated STAT3 (PIAS3) protein promotes SUMOylation and nuclear sequestration of the intracellular domain of ErbB4 protein. J. Biol. Chem. 287, 23216-23226. https://doi.org/10.1074/jbc. M111.335927. 
Tatham, M.H., Geoffroy, M.C., Shen, L., Plechanovova, A., Hattersley, N., Jaffray, E.G., Palvimo, J.J., and Hay, R.T. (2008). RNF4 is a poly-SUMO-specific E3 ubiquitin ligase required for arsenic-induced PML degradation. Nat. Cell Biol. 10, 538-546. https://doi.org/10.1038/ ncb1716.

Terrell, J., Shih, S., Dunn, R., and Hicke, L. (1998). A function for monoubiquitination in the internalization of a G protein-coupled receptor. Mol. Cell 1, 193-202.

Terui, Y., Saad, N., Jia, S., McKeon, F., and Yuan, J. (2004). Dual role of sumoylation in the nuclear localization and transcriptional activation of NFAT1. J. Biol. Chem. 279, 28257-28265. https://doi.org/10.1074/jbc. M403153200.

Tossidou, I., Niedenthal, R., Klaus, M., Teng, B., Worthmann, K., King, B.L., Peterson, K.J., Haller, H., and Schiffer, M. (2012). CD2AP regulates SUMOylation of CIN85 in podocytes. Mol. Cell. Biol. 32, 1068-1079. https://doi. org/10.1128/MCB.06106-11.

Trotman, L.C., Wang, X., Alimonti, A., Chen, Z., TeruyaFeldstein, J., Yang, H., Pavietich, N.P., Carver, B.S., Cordon-Cardo, C., Erdjument-Bromage, H., et al. (2007). Ubiquitination regulates PTEN nuclear import and tumor suppression. Cell 128, 141-156.

Truong, K., Lee, T.D., Li, B., and Chen, Y. (2012). Sumoylation of SAE2 C terminus regulates SAE nuclear localization. J. Biol. Chem. 287, 42611-42619. https:// doi.org/10.1074/jbc.M112.420877.

Ulrich, H.D. (2014). Two-way communications between ubiquitin-like modifiers and DNA. Nat. Struct. Mol. Biol. 21,317-324. https://doi.org/10.1038/nsmb.2805.

Walczak, H., Iwai, K., and Dikic, I. (2012). Generation and physiological roles of linear ubiquitin chains. BMC Biol. 10, 23. https://doi.org/10.1186/1741-7007-10-23.

Wang, L., Wansleeben, C., Zhao, S., Miao, P., Paschen, W., and Yang, W. (2014). SUMO2 is essential while SUMO3 is dispensable for mouse embryonic development. EMBO Rep. 15, 878-885. https://doi.org/10.15252/ embr.201438534.

Wenzel, D.M., and Klevit, R.E. (2012). Following Ariadne's thread: a new perspective on RBR ubiquitin ligases. BMC Biol. 10,24.https://doi.org/10.1186/1741-7007-10-24.
Williamson, A., Werner, A., and Rape, M. (2013). The Colossus of ubiquitylation: decrypting a cellular code. Mol. Cell 49, 591-600. https://doi.org/10.1016/j. molcel.2013.01.028.

Wood, L.D., Irvin, B.J., Nucifora, G., Luce, K.S., and Hiebert, S.W. (2003). Small ubiquitin-like modifier conjugation regulates nuclear export of TEL, a putative tumor suppressor. Proc. Natl. Acad. Sci. U.S.A. 100, $3257-$ 3262. https://doi.org/10.1073/pnas.0637114100.

Wyatt, D., Malik, R., Vesecky, A.C., and Marchese, A. (2011). Small ubiquitin-like modifier modification of arrestin-3 regulates receptor trafficking. J. Biol. Chem. 286, 3884-3893. https://doi.org/10.1074/jbc. M110.152116.

Yokoyama, S., Woods, S.L., Boyle, G.M., Aoude, L.G., MacGregor, S., Zismann, V., Gartside, M., Cust, A.E., Haq, R., Harland, M., et al. (2011). A novel recurrent mutation in MITF predisposes to familial and sporadic melanoma. Nature 480, 99-103. https://doi. org/10.1038/nature 10630 .

Zhang, F.P., Mikkonen, L., Toppari, J., Palvimo, J.J., Thesleff, I., and Jänne, O.A. (2008). Sumo-1 function is dispensable in normal mouse development. Mol. Cell. Biol. 28, 5381-5390. https://doi.org/10.1128/ MCB.00651-08.

Zhong, S., Muller, S., Ronchetti, S., Freemont, P.S., Dejean, A., and Pandolfi, P.P. (2000a). Role of SUMO-1modified PML in nuclear body formation. Blood. 9, 2748-52.

Zhong, S., Salomoni, P., and Pandolfi, P.P. (2000b). The transcriptional role of PML and the nuclear body. Nat. Cell Biol. 2, E85-90. https://doi. org/10.1038/35010583.

Zhou, H.J., Xu, Z., Wang, Z., Zhang, H., Simons, M., and Min, W. (2018). SUMOylation of VEGFR2 regulates its intracellular trafficking and pathological angiogenesis. Nat. Commun. 9, 3303. https://doi.org/10.1038/ s41467-018-05812-2.

Zhu, J., Zhou, J., Peres, L., Riaucoux, F., Honoré, N., Kogan, S., and de Thé, H. (2005). A sumoylation site in PML/ RARA is essential for leukemic transformation. Cancer Cell 7, 143-153. 
